\title{
Anti-Yo-associated autoimmune encephalitis after colon cancer treatment
}

\author{
Na-Yeon Jung, MD, PhD ${ }^{1,2}$; Kyoung-Nam Woo, MD'; Jae Wook Cho, MD, \\ $\mathrm{PhD}^{1,2}$; Hyun-Woo Kim, MD ${ }^{1,2}$ \\ 'Department of Neurology, Pusan National University Yangsan Hospital, Pusan National \\ University School of Medicine, Yangsan, Republic of Korea \\ ${ }^{2}$ Research Institute for Convergence of Biomedical Science and Technology, Pusan National \\ University Yangsan Hospital, Yangsan, Republic of Korea
}

\section{Journal of Neurocritical Care \\ CASE REPORT \\ Received: July 6, 2020 \\ Revised: August 12, 2020 \\ Accepted: August 14, 2020 \\ Corresponding Author: $\mathrm{Na}$-Yeon Jung, MD, PhD Department of Neurology, Pusan National University Yangsan Hospital, Pusan National University School of Medicine and Research Institute for Convergence of Biomedical Science and Technology, 20 Geumo-ro, Mulgeum-eup, Yangsan 50612, Korea Tel: +82-55-360-2122 \\ Fax: +82-55-360-2152 \\ E-mail: nyjung@pusan.ac.kr}

Background: Anti-Yo antibodies are classically associated with paraneoplastic cerebellar degeneration in ovarian and breast cancers and are rarely seen in colon cancer. Anti-Yo-associated paraneoplastic autoimmune encephalitis in colon cancer is rare.

Case Report: A 66-year-old man presented with new-onset seizures after completion of a scheduled treatment for colon cancer. Magnetic resonance imaging showed hyperintense signals with enhancement in the left temporal lobe and insular cortex. Cerebrospinal fluid findings included pleocytosis and elevated protein levels, while Yo antibodies were detected in the serum. There was no relapse of colon cancer, nor were any new cancers found. The patient's symptoms and laboratory test results improved after the administration of high-dose steroids, intravenous immunoglobulin, and plasmapheresis with antiepileptic drugs.

Conclusion: This is a rare case of autoimmune encephalitis with anti-Yo antibodies. The appearance of new-onset seizures during the treatment of malignancy should raise suspicions for paraneoplastic autoimmune encephalitis, even after surgery and chemotherapy are completed.

Keywords: Anti-Yo antibody; Autoimmune encephalitis; Colon cancer; Seizure

\section{INTRODUCTION}

Paraneoplastic neurological syndromes occur because of immune-mediated neuronal dysfunction secondary to systemic malignancy, and the symptoms usually occur before the malignancy is discovered. Anti-Yo antibody is known to be an onconeural antibody that causes paraneoplastic cerebellar degeneration with ataxia and cerebellar dysfunction [1]. Here, we have reported a rare case of autoimmune encephalitis with anti-Yo antibodies presenting with seizures after scheduled treatment for rectosigmoid colon cancer.

\section{CASE REPORT}

A 66-year-old man presented with seizures and altered mental sta-

(C) 2020 The Korean Neurocritical Care Society

This is an Open Access article distributed under the terms of the Creative Commons Attribution Non-Commercial License (http://creativecommons.org/licenses/by-nc/4.0/) which permits unrestricted noncommercial use, distribution, and reproduction in any medium, provided the original work is properly cited. 
tus. He was previously diagnosed with stage IIIB rectosigmoid colon cancer (adenocarcinoma) and underwent treatment with laparoscopic ultra-low anterior resection of the rectum and sigmoid colon 8 months prior. Three weeks prior to consultation, the patient had successfully finished eight cycles of chemotherapy with capecitabine/oxaliplatin. Two days prior to consultation, he was noted to have somnolence. His family was not aware of any memory deficits or personality changes before the seizure. On examination, the patient was febrile $\left(38.5^{\circ} \mathrm{C}\right)$, stuporous, and experiencing generalized tonic-clonic seizures. Brain magnetic resonance imaging (MRI) showed hyperintense signals with enhancement in the left temporal lobe and insular cortex (Fig. 1A). Analysis of the cerebrospinal fluid (CSF) showed pleocytosis (white blood cell [WBC] count, $212 / \mu \mathrm{L}$; lymphocyte, 96.1\%) and elevated protein levels $(72.3 \mathrm{mg} / \mathrm{dL})$, but no malignant cells. Electroencephalography (EEG) revealed periodic lateralized epileptiform discharges originating from the left frontotemporal area. The patient was administered intravenous (IV) acyclovir $(10 \mathrm{mg} / \mathrm{kg} \mathrm{ev-}$ ery 8 hours) and IV levetiracetam (3,000 mg/day), but no clinical improvement was noted. Hence, the patient was administered dexamethasone (20 mg/day), following which his fever and ability to obey commands improved. After he became mentally alert, he became very talkative, but speech content was incongruous. Anti-Yo antibodies were subsequently detected in the patient's serum, but other paraneoplastic and autoimmune antibodies $(\mathrm{Hu}$, Ri, amphiphysin, CV2, Ma2, recoverin, SOX1, titin, NMDA, AMPA, DPPX, LGI1, CASPR2, and GABA-B) were absent, and laboratory tests for infectious agents revealed negative results. $\mathrm{Al}$ though the CSF findings (WBC count, $14 / \mu \mathrm{L}$; protein level, 63.0 $\mathrm{mg} / \mathrm{dL}$ ) began improving on day 14 after onset, the patient's electrographic seizures persisted. We then administered IV immunoglobulin (IVIG) $(400 \mathrm{mg} / \mathrm{kg} /$ day) for 5 days and added valproate (1,800 mg/day) and lacosamide (200 mg/day). On day 21 after onset, he could name and recognize his wife, son, and other relatives. On day 28 after onset, follow-up MRI showed additional hyperintense signals in the left subcallosal gyrus and increased gy-

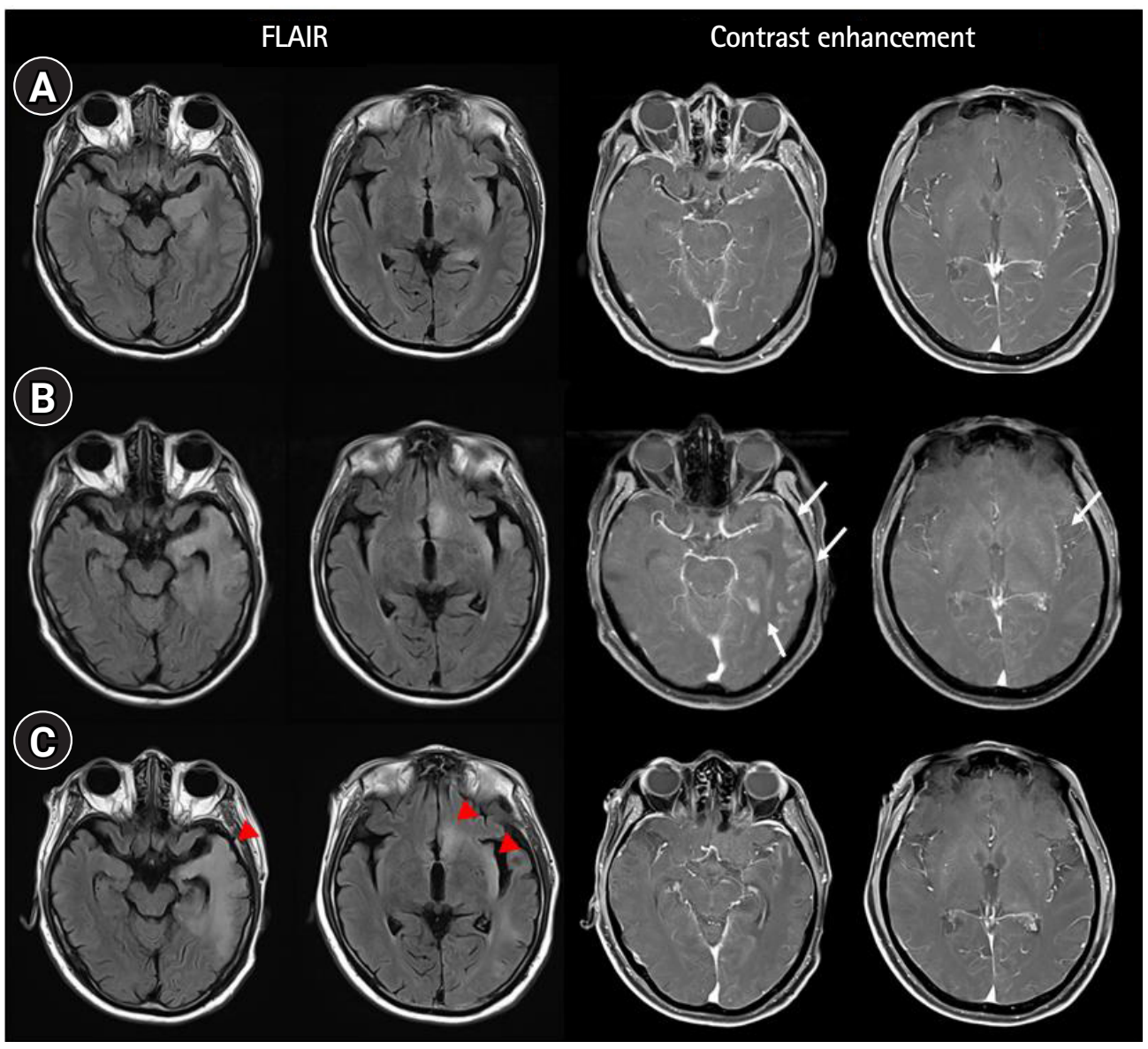

Fig. 1. Fluid-attenuated inversion recovery (FLAIR; left) and contrast-enhanced (right) magnetic resonance imaging taken on the day of onset of the seizure (A), day 28 (B), and day 53 (C). Enhancement in the left temporal lobe and insular cortex (arrows in B) decreased in images of day 53 (C). The last follow-up FLAIR images showed hyperintense signals in the left temporal lobe, subcallosal gyrus, and insular cortex (arrowheads). 
ral enhancement in the left temporal lobe (Fig. 1B). However, the patient's CSF findings (WBC count, $13 / \mu \mathrm{L}$; protein level, 46.7 $\mathrm{mg} / \mathrm{dL}$ ) improved slightly. On day 36 after onset, after follow-up EEG showed no epileptiform discharges, and so the patient was discharged. He had an alert mental state but suffered from cognitive impairment including short-term memory loss, naming difficulty, and decreased verbal comprehension. Korean version of the Mini Mental State Examination was administered, but it was incomplete $(5 / 30)$ because of decreased comprehension.

The patient was readmitted after 2 weeks because of aggression and agitation. His CSF WBC count was $16 / \mu \mathrm{L}$ and CSF protein level was within the normal range. MRI showed hyperintense signals in similar areas as the previous imaging result, but with decreased extent of enhancement (Fig. 1C). EEG revealed periodic lateralized epileptiform discharges in the left parietooccipital area. Anti-Yo antibodies were detected again in the patient's serum. We performed a total of five plasma exchanges and added perampanel $(6 \mathrm{mg} /$ day). We reduced the administration of levetiracetam and ultimately discontinued it. Although the patient still experienced cognitive impairment, poor sleep, and agitation, these were managed by antipsychotic drugs and the patient was able to walk and live at home. Given the improvements in CSF, EEG findings, and the reduction in enhancement on MRI, we did not add other immunosuppressive agents. To determine the possibility of colon cancer relapse or the appearance of a completely new type of cancer, we performed whole-body $18 \mathrm{~F}$-fluorodeoxyglucose-positron emission tomography 2 months after the onset of encephalitis. However, no hypermetabolic lesions were detected. Computed tomography of the chest, abdomen, and pelvis did not show any cancer recurrence or metastasis at the time of symptom onset and after 3 months.

\section{DISCUSSION}

In the present case, rapid progression of altered mental status, cognitive impairment and psychiatric symptoms, new-onset seizure, CSF pleocytosis, and encephalitis on MRI fulfilled the diagnostic criteria for autoimmune encephalitis [2]. Our patient represents a rare case of paraneoplastic autoimmune encephalitis with anti-Yo antibodies associated with colon adenocarcinoma. Most cases of paraneoplastic encephalitis are associated with small cell lung cancer and testicular cancer [3]. Colon cancer has been primarily reported in association with other paraneoplastic syndromes such as subacute cerebellar degeneration and stiff person syndrome $[4,5]$. In addition, anti-Yo antibodies target Purkinje cells and are associated with paraneoplastic cerebellar degeneration [1]. Cancers associated with anti-Yo-antibodies include ovar- ian, uterine, and breast cancers $[1,6]$.

Initially, we considered the possibility of false-positive results with the anti-Yo antibody assay because the anti-Yo antibody did not fit the clinical presentation of the patient. However, it is reasonable to suggest a case of paraneoplastic autoimmune encephalitis because our patient had a history of recent cancer and showed improvement in symptoms and laboratory test results after the administration of high-dose steroids, IVIG, and plasmapheresis [7]. Furthermore, anti-Yo-associated autoimmune encephalitis has been rarely reported [8-10]; thus, clinical heterogeneity of anti-Yo antibody-associated paraneoplastic neurological syndromes needs to be considered. However, because almost $30 \%-50 \%$ of patients with paraneoplastic neurological syndromes do not have any of the well-characterized onconeural antibodies, we could not exclude the existence of other unknown antibodies $[3,11]$.

The general prognosis of patients with autoimmune encephalitis associated with anti-Yo antibodies has not been well described because of its rarity. A previously reported case of autoimmune encephalitis associated with anti-Yo antibodies showed poor prognosis and remained in a permanent vegetative state [9]. The patient was diagnosed with colon adenocarcinoma and underwent subtotal colectomy after the onset of encephalitis. Active immunotherapy needs to be considered in patients with paraneoplastic autoimmune encephalitis associated with anti-Yo antibodies even after surgery and chemotherapy for cancer. Successful cancer treatment does not guarantee the prevention or neurologic improvement of paraneoplastic neurologic syndromes [3].

In $70 \%-80 \%$ of cases, neurological paraneoplastic syndrome antedates the diagnosis of cancer $[1,11,12]$. However, in a few cases, paraneoplastic autoimmune encephalitis occurs during chemotherapy or even after 9 years of treatment $[12,13]$. In our patient, the recurrence of colon cancer or the appearance of a new cancer was not observed after presentation with encephalitis. However, because we could not exclude the possibility of occult cancer, we will closely observe his clinical course. The prevalence of paraneoplastic autoimmune encephalitis after the underlying cancer is considered in remission remains unclear. Therefore, the appearance of new-onset seizures associated with a treated malignancy should raise suspicion for paraneoplastic autoimmune encephalitis as well as brain metastasis, leptomeningeal disease, infectious encephalitis, and metabolic causes. Thus, clinicians should test for paraneoplastic antibodies.

In conclusion, this is a rare case of autoimmune encephalitis with anti-Yo antibodies. The appearance of new-onset seizures during treatment for malignancy should raise suspicions for paraneoplastic autoimmune encephalitis, even after surgery and chemotherapy are completed. 


\section{ARTICLE INFORMATION}

\section{Ethics statement}

This case was approved by the Institutional Review Board of the Pusan National University Yangsan Hospital (IRB No. 05-2020127) and exempted from informed consent.

\section{Conflict of interest}

No potential conflict of interest relevant to this article.

\section{ORCID}

Na-Yeon Jung https://orcid.org/0000-0002-8265-9508

Kyoung-Nam Woo https://orcid.org/0000-0001-8115-0905 Jae Wook Cho https://orcid.org/0000-0002-2742-9136

Hyun-Woo Kim https://orcid.org/0000-0003-1653-7737

\section{Author contributions}

Conceptualization: NYJ. Investigation: KNW, HWK. Writingoriginal draft: NYJ. Writing-review \& editing: NYJ, HWK, JWC.

\section{REFERENCES}

1. Darnell RB, Posner JB. Paraneoplastic syndromes involving the nervous system. N Engl J Med 2003;349:1543-54.

2. Graus F, Titulaer MJ, Balu R, Benseler S, Bien CG, Cellucci T, et al. A clinical approach to diagnosis of autoimmune encephalitis. Lancet Neurol 2016;15:391-404.

3. Pelosof LC, Gerber DE. Paraneoplastic syndromes: an approach to diagnosis and treatment. Mayo Clin Proc 2010;85: 838-54.

4. Martel S, De Angelis F, Lapointe E, Larue S, Speranza G. Paraneoplastic neurologic syndromes: clinical presentation and management. Curr Probl Cancer 2014;38:115-34.
5. Gultekin SH, Rosenfeld MR, Voltz R, Eichen J, Posner JB, Dalmau J. Paraneoplastic limbic encephalitis: neurological symptoms, immunological findings and tumour association in 50 patients. Brain 2000;123(Pt 7):1481-94.

6. Leypoldt F, Wandinger KP. Paraneoplastic neurological syndromes. Clin Exp Immunol 2014;175:336-48.

7. Ebright MJ, Li SH, Reynolds E, Burke JF, Claytor BR, Grisold A, et al. Unintended consequences of Mayo paraneoplastic evaluations. Neurology 2018;91:e2057-66.

8. Seluk L, Taliansky A, Yonath H, Gilburd B, Amital H, Shoenfeld $\mathrm{Y}$, et al. A large screen for paraneoplastic neurological autoantibodies; diagnosis and predictive values. Clin Immunol 2019; 199:29-36.

9. Adam VN, Budinčević $H$, Mršić V, Stojčić EG, Matolić M, Markić A. Paraneoplastic limbic encephalitis in a patient with adenocarcinoma of the colon: a case report. J Clin Anesth 2013; 25:491-5.

10. Berger B, Bischler P, Dersch R, Hottenrott T, Rauer S, Stich O. "Non-classical" paraneoplastic neurological syndromes associated with well-characterized antineuronal antibodies as compared to "classical" syndromes: more frequent than expected. J Neurol Sci 2015;352:58-61.

11. Said S, Cooper CJ, Reyna E, Alkhateeb H, Diaz J, Nahleh Z. Paraneoplastic limbic encephalitis, an uncommon presentation of a common cancer: case report and discussion. Am J Case Rep 2013;14:391-4.

12. Attademo L, De Falco S, Rosanova M, Esposito M, Mazio F, Foschini F, et al. A case report of limbic encephalitis in a metastatic colon cancer patient during first-line bevacizumab-combined chemotherapy. Medicine (Baltimore) 2018;97:e0011.

13. Silsby M, Clarke CJ, Lee K, Sharpe D. Anti-Hu limbic encephalitis preceding the appearance of mediastinal germinoma by 9 years. Neurol Neuroimmunol Neuroinflamm 2020;7:e685. 